\title{
Análise comparativa de dois métodos de proteção miocárdica em pacientes de alto risco para cirurgia de revascularização do miocárdio
}

\author{
Fernando Antônio Roquete REIS FILHO*, Luiz Cláudio Moreira LIMA*, Giancarlo Grossi MOTA*, \\ Leonardo A. GONÇALVES ${ }^{\star}$, Maurício C. GOMES*, Rodrigo de Castro BERNARDES*, \\ Raul Corrêa RABELO*
}

RBCCV 44205-315

Reis Filho F A R, Lima C M, Mota G G, Gonçalves L A, Gomes M C, Bernardes R C, Rabelo R C - Análise comparativa de dois métodos de proteção miocárdica em pacientes de alto risco para cirurgia de revascularizaçāo do miocárdio. Rev Bras Cir Cardiovasc 1996; 11 (4): 238-47.

RESUMO:Objetivo: Comparar a tradicional proteção miocárdica oferecida pela cardioplegia cristalóide gelada infundida na aorta associada à hipotermia sistêmica e ao pinçamento intermitente da aorta(Grupol), à cardioplegia sangūinea normotérmica com induçāo pela aorta e manutenção por via retrógrada (seio coronário) associada a normotermia sistêmica.

Casuística e Métodos: No período de maio de 1992 a maio de 1994, foram selecionados de forma consecutiva, não randomizada, de acordo com pré-requisitos que acrescentavam alta morbi-mortalidade à cirurgia de revascularização miocárdica (CRVM), dois grupos de 50 pacientes. Todos os pacientes selecionados apresentavam: instabilidade ou intratabilidade clínica, mais de três ramos arteriais coronários, ou tronco de coronária esquerda ocluídos, ou gravemente obstruídos e grave disfunção ventricular esquerda de etiologia isquêmica.

Resultados: Os grupos foram semelhantes quanto às variáveis pré-operatórias. OGrupoll teve maior média de enxertos $(3,82 \times 3,40)$, conseqūentemente maior tempo de pinçamento aórtico $(70,74 \times 62,6 \mathrm{~min})$ e maior tempo de circulação extracorpórea (CEC) $(97,50 \times 93,86 \mathrm{~min})$. O Grupo I teve maior incidência de fibrilaçăo ventricular após despinçamento aórtico $(20 \% \times 4 \%) p=0,032$, maior necessidade de inotrópicos para sair da CEC $(18 \% \times 12 \%)$ maior necessidade de assistência circulatória (14\%×8\%), maior incidência de infarto agudo do miocárdio (20\%×12\%); teve ainda menor incidência de vetilação mecânica prolongada $(6 \% \times 12 \%)$, maior mortalidade imediata $(12 \% \times 6 \%)$, hospitalar $(14 \% \times 10 \%)$ e global $(26 \% \times 16 \%)$ e maior incidência de óbitos de causas cardiacas $(12 \% \times 4 \%)$.

Conclusăo: A comparabilidade dos grupos ficou prejudicada pela maior média de enxertos doGrupo II, conferindo maior gravidade a este grupo. Mesmo assim, os resultados deste grupo foram superiores aos do Grupo I, embora isto nāo tenha sido corroborado pela análise estatística. Estes resultados, associados à impressão clínica, o aprimoramento técnico e o reconhecimento das limitaçōes do método nos obrigaram a adotá-lo como forma de proteção miocárdica neste grupo específico de pacientes.

DESCRITORES: Parada cardiaca induzida, métodos. Revascularização miocárdica, fatores de risco. Revascularizaçāo miocárdica, estudo comparativo.

\footnotetext{
Trabalho realizado no Instituto do Coração do Hospital Madre Tereza. Belo Horizonte, MG, Brasil. Apresentado ao $23^{\circ}$ Congresso Nacional de Cirurgia Cardiaca. Recife, PE, 20 a 23 de março, 1996.

* Do Instituto do Coração do Hospital Madre Tereza.

Endereço para correspondência: Fernando Antônio Reis Filho. Av. Raja Gabaglia, 1002-Gutierrez, Belo Horizonte, MG, Brasil. CEP: 30380-090. Tel. 337-5933.
} 


\section{INTRODUÇÃO}

O desenvolvimento de métodos de proteção miocárdica vem caminhando lado a lado com os avanços da cirurgia cardíaca. Desde as primeiras comunicaçōes sobre soluções cardioplégicas para permitir a parada cardíaca eletiva (1), várias técnicas têm surgido e desaparecido. Na última década, houve grande interesse sobre a via de liberação de soluções cardioplégicas, assim como o veículo e a composição destas soluções.

O conceito de perfusão cardíaca retrógrada não é novo. Estudos iniciados por BLANCO et al. (2) em 1956 sugeriam a possibilidade de utilizar a via retrógrada (seio coronário) para proteção do miocárdio durante o pinçamento aórtico, para o tratamento de lesōes da valva aórtica. Esta abordagem foi posteriormente utilizada com sucesso, tanto experimentalmente por GOTT et al. (3), quanto clinicamente por LILLEHEY et al. (4).

Nos últimos anos, a perfusão cardíaca retrógrada (retroplegia) vem novamente chamando a atenção dos cirurgiões, como forma de proteger o miocárdio. Os estudos de SOLORZANO et al (5) e, posteriormente, MENASCHÉ et al (6), demonstraram a viabilidade da utilização da retroplegia para proteger o miocárdio e, ainda, evitar a lesão dos óstios coronários na operação da valva aórtica. Desde então, vários trabalhos têm sido publicados mostrando um crescente interesse nesta técnica $(7-11)$.

Entretanto, a despeito de um grande número de evidências experimentais $(12-17)$, que demonstram a superioridade da proteção miocárdiça por via retrógrada sobre a proteção miocárdica por via anterógrada, poucas evidências clínicas têm sido descritas. Talvez porque os estudos experimentais falhem em reproduzir com fidelidade as condições clínicas e anatômicas encontradas na doença coronariana humana, como, por exemplo, a presença de colaterais e as diferenças anatômicas do sistema venoso cardiaco humano e o animal. Da mesma forma, os estudos clínicos (18-20) não levam em conta a gravidade das lesões coronarianas ou o grau de disfunçāo ventricular, freqüentemente comparando populaçōes heterogêneas ou de baixo risco cirúrgico, o que dificulta melhor avaliação dos métodos de proteção empregados.

Paralelamente a estes estudos, em 1991 LICHTENSTEIN et al. (21) questionavam os supostos benefícios da hipotermia como proteção sistêmica e cardíaca, tendo em vista os efeitos indesejáveis da hipotermia sobre a função enzimática, a estabilidade da membrana, o seqüestro de cálcio, a utilização de glicose, a geração e utilização de ATP e a extração de oxigênio, assim como no pH e home- ostasia oncótica. Defendiam que a manutenção de um meio normotérmico oxigenado, associado à manutenção da parada eletromecânica do coração, conservaria a homeostasia e, conseqüentemente, a estrutura das células miocárdicas.

Desta forma, este estudo foi idealizado com o intuito de comparar dois métodos de proteção miocárdica: a clássica cardioplegia cristalóide gelada (tipo Saint Thomas) infundida na aorta, associada a pinçamento intermitente e a hipotermia tópica e sistêmica moderada - doravante designada anterógrada - e o novo conceito a cardioplegia sangüínea normotérmica com indução via anterógrada e manutenção por via retrógrada associada à normotermia sistêmica - doravante designada retrógrada como preconizado por LICHTENSTEIN et al (21), BUCKBERG (22), KALMBACH \& BHAYANA (23)', DRINKWATER et al. $(24,25)$

Para maior confiabilidade do estudo, testamos a hipótese de um método ser superior ao outro, em pacientes considerados de alta morbi-mortalidade para a operação revascularização do miocárdio.

\section{CASUISTICA E MÉTODOS}

\section{População}

Dos 689 pacientes submetidos a tratamento cirúrgico de revascularização do miocárdio no período de maio de 1992 a maio de 1994 , foram selecionados de forma consecutiva, não randomizada, 50 pacientes (de 350 pacientes que utilizaram a cardioplegia anterógrada (Grupo I) e 50 pacientes (de 339 pacientes que utilizaram a cardioplegia retrógrada (Grupo II). Os critérios de seleção, considerados pré-requisitos, se basearam na existência de fatores que acrescentassem alta mortalidade ao tratamento cirúrgico, até $30 \%$ pelo estudo do CASS (26). Todos os pacientes selecionados se enquadaram em todos os pré-requisitos.

\section{Pré-Requisitos para Seleção}

1) Critérios clínicos: angina classe IV (definida pela Canadian Cardiology Society), angina instável (definida como a refratariedade ao tratamento clínico antianginoso intensivo e em regime hospitalar), angina pós-infarto (ocorrência de angina na fase hospitalar de tratamento do infarto agudo do miocárdio) e angina pós trombolitico (ocorrência de angina na fase hospitalar de tratamento do infarto agudo do miocárdio após reperfusão química utilizando trombolíticos). 
Reis Filho FA R, Lima C M, Mota G G, Gonçalves LA, Gomes M C, Bernardes R C, Rabelo R C - Análise comparativa de dois métodos de proteçāo miocárdica em pacientes de alto risco para cirurgia de revascularizaçāo do miocárdio. Rev Bras Cir Cardiovasc $1996 ; 11(4): 238-47$.

2) Critérios anatômicos: tri-arterial (três ou mais ramos coronarianos ocluídos ou gravemente obstruídos; sub-ocluidos = presença de lesão obstrutiva maior que $70 \%$ ); lesão de tronco de coronária esquerda (obstrução maior que $50 \%$ do tronco da artéria coronária esquerda)

3) Critérios funcionais: presença de grande disfunção ventricular de etiologia isquêmica (definida como fração de ejeção menor que $30 \%$ avaliada pela planigrafia da ventriculografia esquerda em projeção obliqua anterior direita).

Obs: Todos os pacientes obedeceram os três critérios.

\section{Variáveis e Definiçōes} foram:

As variáveis pré e trans-operatórias analisadas

- Idade.

- Sexo.

- Peso.

- Número de enxertos realizados (pontes de veia safena e/ou anastomoses de artéria torácica interna).

- Tempo de pinçamento aórtico.

- Tempo de circulação extracorpórea.

- Quantidade de solução cardioplégica utilizada.

As variáveis pós-circulação extracorpórea (CEC) analisadas foram:

- Recuperação do ritmo cardíaco (incidência de fibrilação ventricular): recuperação espontânea ou não dos batimentos cardíacos, sem a necessidade de estimulação artificial externa, drogas cronotrópicas positivas ou desfibrilação elétrica, após 3 minutos de despinçamento aórtico.

- Alargamento do QRS: medida eletrocardiográfica da duração do complexo QRS antes e depois da operação, considerando anormal um aumento maior que $0,4 \mathrm{mseg}$ em relação ao ECG basal.

- Necessidade de inotrópicos pós-CEC: utilização de qualquer amina vasoativa para auxiliar na saída de CEC.

- Necessidade de assistência circulatória: manutenção da CEC ou utilização de circuito "átrio esquerdo-aorta" por período superior a $30 \mathrm{mi}$ nutos após o despinçamento aórtico, ou a utilização de balão intra-aórtico (BIA) para saída de CEC e/ou suporte pós-operatório.

As variáveis analisadas no pós-operatório, na Unidade de Tratamento Intensivo, foram:
- Necessidade de inotrópicos no pós-operatório: utilização de qualquer amina vasoativa em doses inotrópicas e/ou vasoconstritoras.

- Ocorrência de infarto agudo do miocárdio (IAM): definido como o surgimento de "novas" ondas $Q$ no eletrocardiograma, alterações enzimáticas (CK-MB) e/ou aparecimento de zonas acinéticas na análise comparativa ecocardiográfica.

- Tempo de ventilação mecânica.

- Incidência de ventilação mecânica prolongada: definida como a manutenção da ventilação mecânica por período superior a 24 horas.

- Tempo de permanência no CTI (Centro de Tratamento Intensivo pós-operatório).

- Tempo de internação hospitalar.

- Mortalidade imediata: ocorrência de óbitos nas primeiras 48 horas de pós-operatório.

- Mortalidade hospitalar: ocorrência de óbitos na fase de internação hospitalar ou nos primeiros 30 dias de pós-operatório.

- Óbitos de causa cardíaca: causados por síndrome de baixo débito cardiaco, insuficiência cardíaca congestiva e/ou arritmias refratárias.

- Óbitos de causas não cardíacas: quando relacionados diretamente a qualquer outra doença e não ao aparelho cardiovascular.

\section{TÉCNICA CIRÚRGICA}

Todos os pacientes foram submetidos à revascularização do miocárdio sob anestesia geral balanceada (inalatória + venosa), com ventilação mecânica controlada ciclada a volume $(10 \mathrm{ml} / \mathrm{kg})$, com oxigênio a $100 \%$.

Os pacientes foram submetidos a esternotomia mediana, com dissecção e preparo de enxertos venosos (ou arteriais). Instituição de circuito extracorpóreo por canulação de átrio direito via aurícula direita com cânula de duplo estágio e aorta ascendente.

Todos os pacientes foram monitorizados por eletrocardiograma contínuo, pressão intra-arterial e pressão venosa central, débito urinário contínuo. A monitorização com cateter de Swan-Ganz foi realizada apenas naqueles pacientes com dificuldade de saída da circulação extracorpórea e/ou em uso de assistência circulatória.

Todos os pacientes tiveram o mediastino e as cavidades pleurais (em caso de abertura das mesmas) drenadas em sistema de selo d'água. Foi implantado eletrodo de marcapasso temporário em VD em todos os pacientes sem distúrbios graves do ritmo, e marcapasso de dupla câmara temporário ( $A D$ e VD) naqueles que cursaram com distúrbios do ritmo. 
Reis Filho FA R, Lima C M, Mota G G, Gonçalves L A, Gomes M C, Bernardes RC, Rabelo R C - Análise comparativa de dois métodos de proteção miocárdica em pacientes de alto risco para cirurgia de revascularizaçāo do miocárdio. Rev Bras Cir Cardiovasc $1996 ; 11(4): 238-47$

\section{Cardioplegia}

\section{Grupo I (anterógrada)}

Realizamos hipotermia sistêmica moderada $\left(28^{\circ} \mathrm{C}\right)$ com posicionamento de um cateter Gelco 14 "French" na aorta ascendente para infusão de solução cardioplégica. A solução cardioplégica tipo Saint Thomas $\left(\mathrm{a}^{\circ} \mathrm{C}\right)$ era infundida na aorta ascendente, após pinçamento, na proporção de $500 \mathrm{ml}$ para cada período de pinçamento (25 $\mathrm{min}$ a $30 \mathrm{~min}$ ). Foram permitidos períodos de despinçamento de 5 minutos.

\section{Grupo II (retrógrada)}

Posicionamento de cateter Gelco 14 "French" na aorta ascendente. Feitura de sutura em bolsa no átrio direito abaixo da cânula venosa e posicionamento por palpação de cânula de retroplegia (DLP - RCSP Cannula with cuff 15F - DLP Inc., Grand Rapids, MI, USA) no seio coronário. A técnica de infusão baseou-se nos princípios preconizados por LICHTENSTEIN et al. (21), BUCKBERG (22), KALMBACH \& BHAYANA (23), DRINKWATER et al. (24. 25), constando de indução anterógrada e manutenção intermitente retrógrada, de solução cardioplégica sangüínea normotérmica, conseguida por mistura do sangue arterializado do reservatório do oxigenador com a solução cardioplégica, na proporção de duas partes de sangue para uma parte de solução cardioplégica.

Utilizamos sistema de reservatório e tubos para cardioplegia da DMG Equipamentos Médicos Ltda., constando de sistema de tubos e conexões que permitiam realização da mistura e $a$ infusão controlada da solução cardioplégica, nas proporções e tempos desejados.

A indução foi realizada por infusão de solução cardioplégica no ínicio da aorta, limitada por pressão (máximo de $120 \mathrm{mmHg}$ ) após pinçamento da porção ascendente, até se alcançar completa assistolia cardíaca. Desviava-se, então, através de um tubo em $\mathrm{Y}$, a infusão da solução para a cânula de retroplegia. Após alcançada assistolia, a infusão por via retrógrada era apenas de sangue desviado do reservatório arterial do sistema extracorpóreo, sem a adição da solução cardioplégica. Esta era adicionada quando o coração esboçava alguma atividade mecânica ou elétrica. A infusão por via retrógrada foi realizada de forma intermitente, já que, durante a feitura das anastomoses distais, era eventual e temporariamente interrompida para permitir um campo cirúrgico exangue (o tempo médio de infusão correspondeu a $69 \%$ do tempo total de pinçamento aórtico). A infusão por via retrógrada era controlada por volume (250 a $300 \mathrm{ml} / \mathrm{min}$ ) e limitada por pressão (pressão máxima permitida de $40 \mathrm{mmHg}$ ). Pres- sões muito elevadas indicavam excessiva introdução da cânula no seio coronário e, muito baixas, seu deslocamento anterior em direção ao aórtico direito, condições que impunham o seu reposicionamento.

A quantidade de solução cardioplégica utilizada está demonstrada no Gráfico 1.

\section{Soluções Cardioplégicas}

\section{Cardioplegia anterógrada}

\section{CARDIOPLEGIA SAINT THOMAS:}

Ringer Simples ................................... 500

Cloreto de Potássio .........................1,197gr

Cloreto de Magnésio ......................3,325mg

Cloridrato de Procaína ....................278,8mg

* Infundida a $4^{\circ} \mathrm{C}$

\section{SOLUÇÃO DE BUCKBERG "MODIFICADA"}

Solução de Glicose a $5 \%$................. $500 \mathrm{ml}$

Cloreto de Potássio ............................ 27mEq

Bicarbonato de Sódio ........................20 $20 \mathrm{mEq}$

* adicionada na proporção 2:1 de sangue

\section{Saída da Circulação Extracorpórea}

Após despinçamento aórtico era permitido um período de até 10 minutos para completa recuperação dos batimentos cardiacos de forma efetiva e satisfatória; caso isto não acontecesse eram instituidas medidas de suporte: uso de drogas vasoativas

GRÁFICO 1

QUANTIDADE DE SOLUÇĀO CARDIOPLÉGICA UTILIZADA (EM MILIMITROS +/-DP)

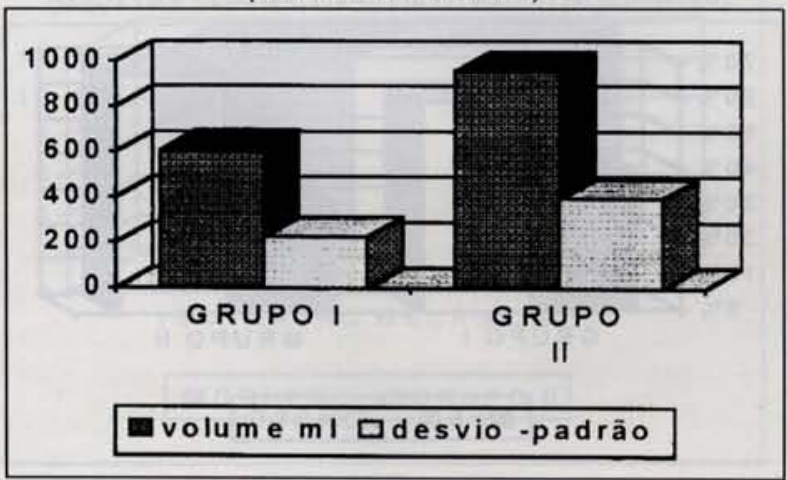


Reis Filho F A R, Lima C M, Mota G G, Gonçalves L A, Gomes M C, Bernardes RC, Rabelo R C - Análise comparativa de dois métodos de proteção miocárdica em pacientes de alto risco para cirurgia de revascularização do miocárdio. Rev Bras Cir Cardiovasc $1996 ; 11(4): 238-47$

(dopamina, dobutamina, adrenalina, noradrenalina, nitroprussiato de sódio, nitroglicerina). Se estas medidas também não alcançassem êxito era instituída assistência circulatória, quer por circuito átrio esquerdo-aorta, ou instalação de contrapulsação com balão intra-aórtico, mantidos até completa estabilização hemodinâmica (medidas hemodinâmicas realizadas com cateter de Swan-Ganz).

A desfibrilação interna foi realizada quando, após 3 minutos de despinçamento aórtico, a fibrilação ventricular ou taquicardia ventricular não se convertia espontaneamente para ritmo sinusal.

\section{Análise Estatística}

Utilizou-se a técnica do Qui quadrado (com correção de Yates) para comparação entre proporçöes ou o Teste Exato de Fischer, quando indicado. Para comparação entre medidas, empregou-se a Análise de Variância. Foi considerado o limite $5 \%$ $(p<0,05)$ para a significância estatística.

A significância estatística está demonstrada no texto, os dados säo apresentados pela média +/desvio padrão (DP) e NS designará não significante, quando não houver significância estatística.

\section{RESULTADOS}

Os dados pré e trans-operatórios estão sumarizados nos Gráficos 2, 3, 4, 5, 6 e 7. Quarenta por cento dos pacientes do Grupo I foram do sexo feminino, contra $32 \%$ no Grupo II. O Grupo I apresentou média de idades de 57,88 anos $(D P=8,63)$ e o Grupo II 57,26 (DP=8,75). O Grupo I apresentou média de peso de $66,32 \mathrm{~kg}(\mathrm{DP}=14,07)$ e o Grupo II $66.02(\mathrm{DP}=8,83)$.

GRÁFICO 2

DISTRIBUIÇĀO QUANTO AO SEXO (EM PERCENTAGEM)

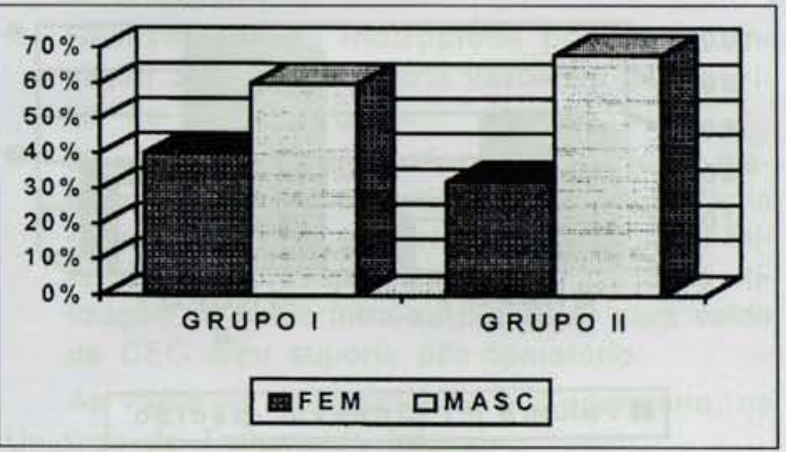

GRÁFICO 3

DISTRIBUIÇĀO QUANTO Aं IDADE

(VALOR EM ANOS +/-DESVIO PADRĂO)

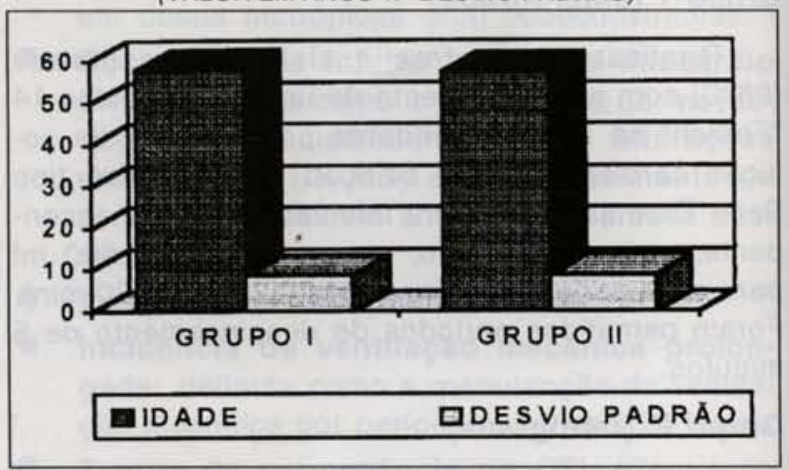

GRÁFICO 4

DISTRIBUIÇÃO QUANTO AO PESO (EM QUILOS)

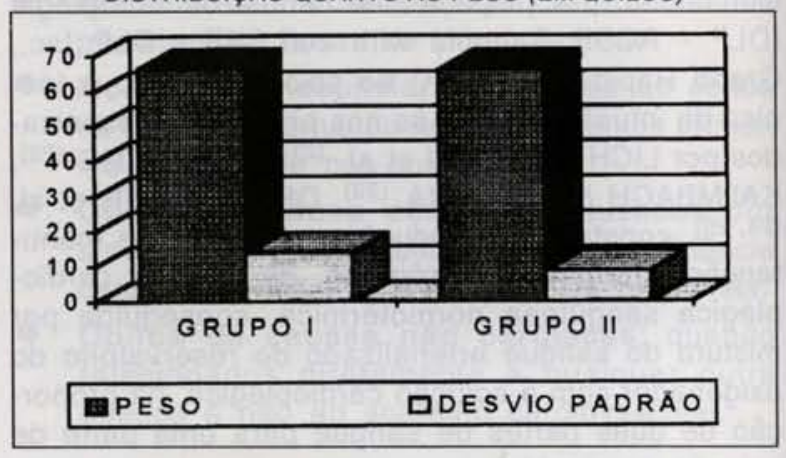

GRÁFICO 5

NÚMERO DE ENXERTOS REALIZADOS

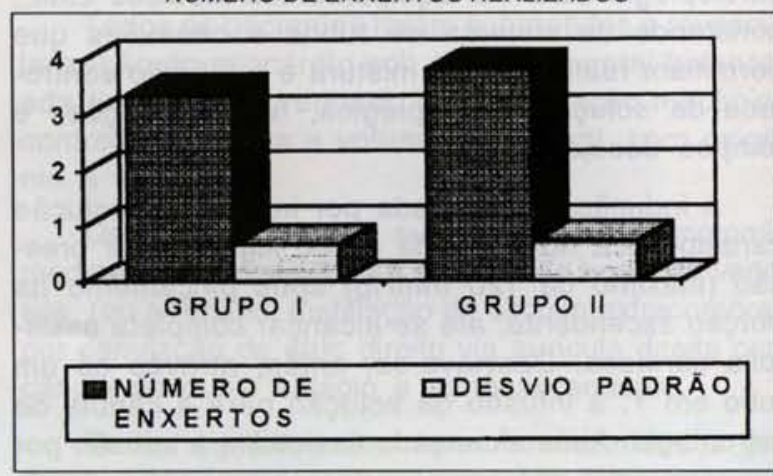

GRÁFICO 6

TEMPO DE PINCAMENTO AÓRTICO (EM MINUTOS)

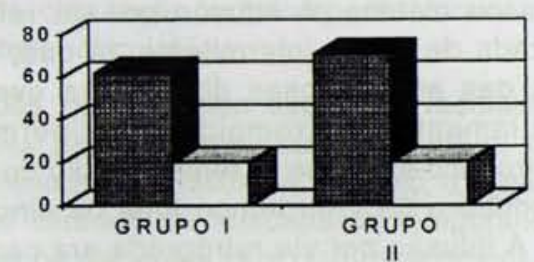

TEMPO DE PINCCAMENTO $\square$ DESVIO PADRÃO 
GRÁFICO 7 TEMPO DE CIRCULAÇÃO EXTRACORPÓREA (EM MINUTOS)

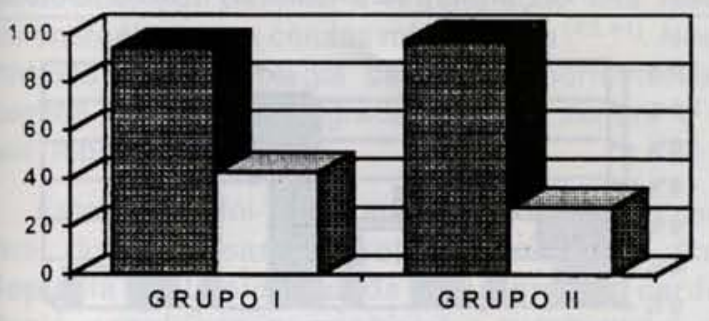

DTEMPO DE CEC DDESVIO PADRĀO

GRÁFICO 8

INCIDÊNCIA DE FIBRILAÇĀO VENTRICULAR (FIBRL), ALARGAMENTO DO QRS (QRS), NECESSIDADE DE INOTRÓPICOS PARA SAIDA DE CEC (INO PÓS CEC) E NECESSIDADE DE BALĀO INTRA-AÓRTICO OU ASSISTENNCIA CIRCULATÓRIA PÓS CEC (BIA OU ASSIS)

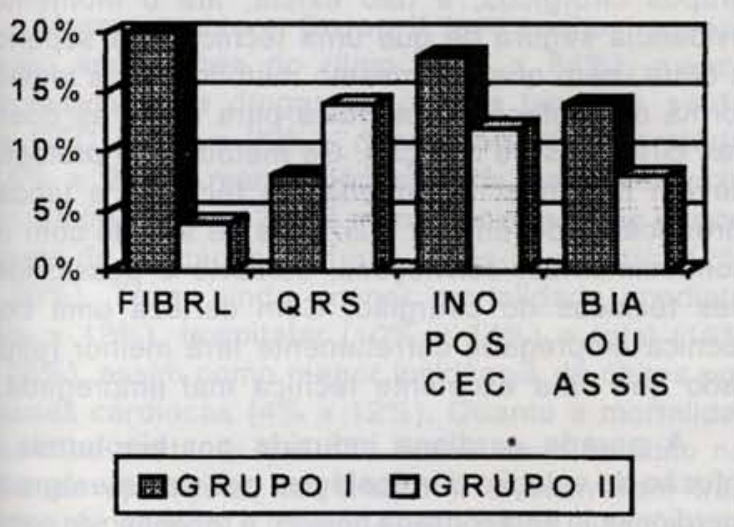

GRÁFICO 9

NECESSIDADE DE DROGAS INOTRÓPICAS NO PÓS-OPERATÓ. RIO (INOT POS - OP) E INCIDENNCIA DE INFARTO AGUDO DO MIOCARDIO (IAM)

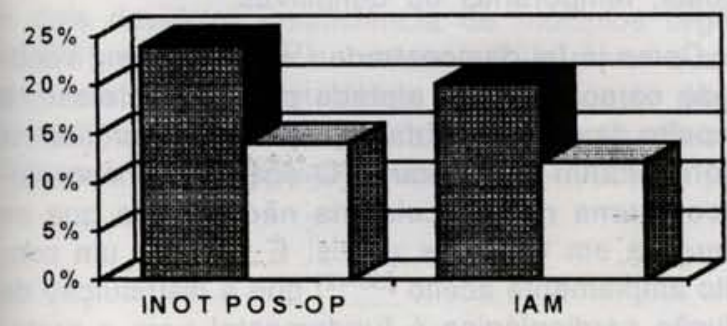

GGRUPOI DGRUPO ॥
O Grupo II teve maior número de enxertos realizados (pontes de safena aorto-coronária + artérias torácicas internas) $3,82(\mathrm{DP}=0,72) \times 3,40$ $(D P=0,67)$. Esta ocorrência contribuiu para que a probabilidade de significância correspondesse a 0,047 , podendo, assim, prejudicar a comparabilidade entre os grupos.

O Grupo I apresentou média de $62,06 \mathrm{~min}$ de pinçamento aórtico $(D P=19,90)$ e $93,86 \mathrm{~min}$ de circulação extracorpórea ( $D P=42,70)$, enquanto o Grupo II apresentou média de $70,74 \mathrm{~min}$ de pinçamento aórtico $(D P=19,97)$ e $97,50 \mathrm{~min}$ de circulação extracorpórea $(\mathrm{DP}=28,23)$.

Os grupos foram semelhantes quanto a idade, sexo e peso. O Grupo II teve maior média de enxertos implantados $(3,80 \times 3,36)$, e maior tempo de pinçamento aórtico e circulação extracorpórea. O Grupo II utilizou maior quantidade de solução cardioplégica, já que a retroplegia contínua, uma vez que oferece um meio aeróbico para o coração, também predispõe um retorno mais precoce da atividade eletromecânica.

Os dados pós-CEC estão sumarizados nos Gráficos 8 e 9. O Grupo I teve maior incidência de fibrilação ventricular após o despinçamento aórtico, que exigiu desfibrilação interna $(20 \% \times 4 \%) p=0,032$, teve menor ocorrência de alargamento do QRS (14\% x $8 \%$ ) NS, maior necessidade de inotrópicos para saída de circulação extracorpórea $(18 \% \times 12 \%)$ NS e maior necessidade de balão intra-aórtico e assistência circulatória $(14 \% \times 8 \%)$ NS.

Os pacientes do Grupo II tiverem menor necessidade de drogas inotrópicas para suporte hemodinâmico no pós-operatório $(14 \% \times 24 \%)$ NS e menor incidência de infarto agudo do miocárdio $(12 \% \times$ $20 \%$ ) NS (Gráfico 9).

Os dados pós-operatórios tardios estão sumarizados nos Gráficos 10,11 e 12 . Estes dados demonstram que os pacientes do Grupo I tiveram

GRÁFICO 10

TEMPO MÉDIO DE VENTILAÇÃO MECÂNICA (EM HORAS)

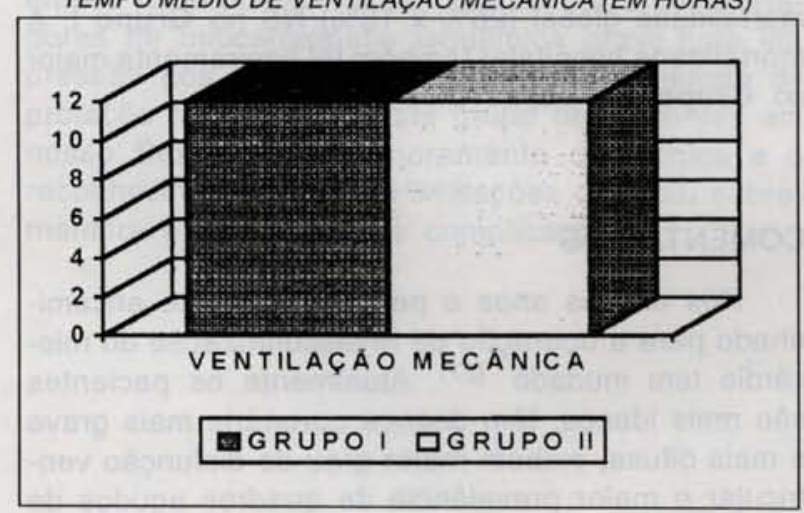


Reis Filho FA R, Lima C M, Mota G G, Gonçalves L A, Gomes M C, Bernardes R C, Rabelo R C - Análise comparativa de dois métodos de proteção miocárdica em pacientes de alto risco para cirurgia de revascularização do miocárdio. Rev Bras Cir Cardiovasc $1996 ; 11(4): 238-47$

GRÁFICO 11

INCIDÊNCIA DE VENTILAÇĀO MECÂNICA PROLONGADA

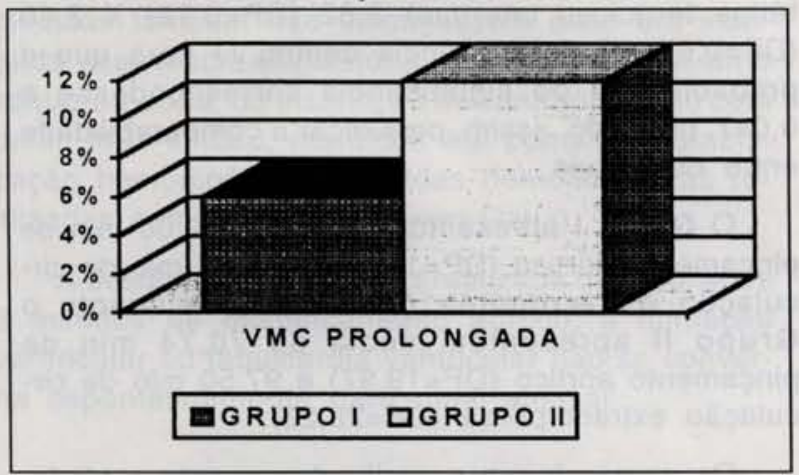

GRÁFICO 12

TEMPO DE INTERNAÇÃO NO CENTRO DE TRATAMENTO INTENSIVO (CTI) E TEMPO DE INTERNAÇĀO HOSPITALAR (HOSP) (EM DIAS)

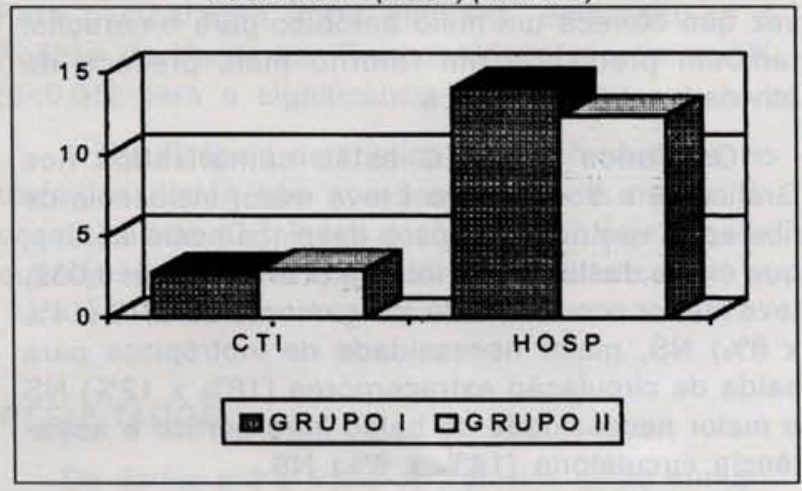

menor incidência de ventilação mecânica prolongada $(6 \% \times 12 \%)$ NS, apesar da quase igualdade nos tempos médios de ventilação mecânica $(12 \times 11,9$ horas) NS. O tempo de CTI (2,7 dias $\times 2,9$ dias) NS e o tempo de internação hospitalar $(14,1$ dias $\times 12,4$ dias) NS foram semelhantes.

Os dados referentes a mortalidade estão sumarizados no Gráfico 13. Estes dados demonstram maior mortalidade imediata $(12 \% \times 6 \%)$ NS e por causas cardiacas $(12 \% \times 4 \%) \mathrm{NS}$, assim como maior mortalidade global $(26 \% \times 16 \%)$ NS no Grupo I. A mortalidade hospitalar também foi ligeiramente maior no Grupo I $(14 \% \times 10 \%)$ NS.

\section{COMENTÁRIOS}

Nos últimos anos o perfil do paciente encaminhado para a operação de revascularização do miocárdio tem mudado (27). Atualmente os pacientes são mais idosos, têm doença coronária mais grave e mais difusa, exibem maior grau de disfunção ventricular e maior prevalência de quadros agudos de
GRÁFICO 13

MORTALIDADE IMEDIATA (IMEDIAT), HOSPITALAR (HOSP), TOTAL (TOTAL) E DE CAUSAS CARDIACAS (CARDIC)

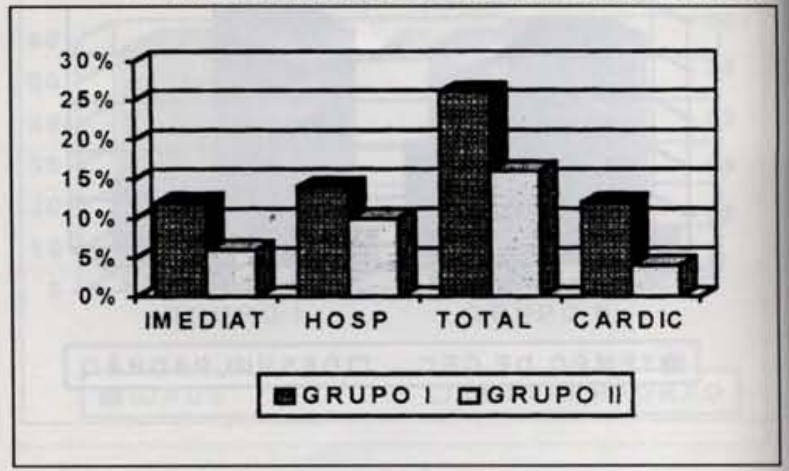

isquemia e/ou infarto agudo do miocárdio. Desta forma, também os métodos de proteção miocárdica têm mudado.

Quase todas as técnicas de proteção miocárdica introduzidas desde os primórdios da cirurgia cardiaca ainda são utilizadas atualmente por um ou mais grupos cirúrgicos, e não existe, até o momento, evidência segura de que uma técnica seja superior a outra, nem que um mesmo método seja a melhor forma de proteção miocárdica para todas as doenças cirúrgicas do coração. Os métodos de proteção devem pertencer ao arsenal de técnicas e táticas cirúrgicas e devem ser utilizados de acordo com os conhecimentos, convicções, conforto e possibilidades técnicas do cirúrgião. Com certeza uma boa técnica empregada corretamente terá melhor resultado que uma excelente técnica mal empregada.

A parada cardíaca induzida por hipotermia $e$ infusão de solução cardioplégica por via anterógrada (cardioplegia anterógrada gelada) é reconhecida como forma segura e eficaz de proteção miocárdica $(28,29)$. Entretanto, existem evidências clínicas e experimentais $(30-39)$ de que, na presença de obstruções coronarianas ocorreria uma distribuição desigual e inadequada da solução cardioplégica, levando a má proteção, principalmente daquelas áreas já mais acometidas pela isquemia, acarretando, conseqüentemente, disfunções miocárdicas regionais ou mesmo globais, temporárias ou definitivas.

Como já foi demonstrado ${ }^{(12)}$, os sistema venoso do coração não é afetado por aterosclerose, a despeito da gravidade da doença aterosclerótica na árvore arterial coronariana. O sistema venoso cardíaco é uma rede circulatória não valvada que se comunica em todos os niveis. É também um conceito amplamente aceito ${ }^{(30-42)}$ que a distribuição da solução cardioplégica é fundamental para a proteção do miocárdio durante a parada cardíaca eletiva e que a doença aterosclerótica coronariana diminui ou impossibilita esta distribuição de forma homogê- 
Reis Filho F A R, Lima C M, Mota G G, Gonçalves LA, Gomes M C, Bernardes R C, Rabelo R C - Análise comparativa de dois métodos de proteção miocárdica em pacientes de alto risco para cirurgia de revascularização do miocárdio. Rev Bras Cir Cardiovasc $1996 ; 11(4): 238-47$.

nea. Além disto, a perfusão normotérmica do coração em assistolia pode melhorar o metabolismo miocárdico, por permitir a regeneração das reservas energéticas das células miocárdicas ${ }^{(43,44)}$. Neste contexto, a cardioplegia sangüínea normotérmica liberada de forma retrógrada é eficaz segura e de fácil reprodutibilidade.

Este estudo foi idealizado com o intuito de comparar duas técnicas de proteção miocárdica (cardioplegia cristalóide gelada anterógrada $X$ cardioplegia sangüínea normotérmica retrógrada) em pacientes com alto risco cirúrgico e mortalidade cirúrgica elevada devido a lesões coronarianas graves, instabilidade ou intratabilidade clínica e disfunção ventricular importante.

Os grupos foram comparáveis quanto a idade, sexo e peso. Houve, entretanto, maior número de enxertos realizados e, conseqüentemente, maior tempo de circulação extracorpórea e maior tempo de pinçamento aórtico nos pacientes do Grupo II, o que pode nos levar a inferir maior gravidade neste grupo.

Mesmo assim, o Grupo II teve melhor recuperação espontânea do ritmo ( $96 \%$ x $84 \%)$, menor necessidade de drogas vasoativas tanto na saída da CEC $(16 \% \times 22 \%)$, quanto no pós-operatório (14\% x 24\%), menor necessidade de assistência circulatória $(8 \% \times 12 \%)$ e menor incidência de infarto agudo do miocárdio no trans e pós-operatório (12\% x 20\%). Teve, ainda, menor mortalidade imediata $(6 \% \times 12 \%)$, hospitalar $(10 \% \times 14 \%)$ e total $(16 \%$ x 26\%), assim como menor incidência de óbitos por causas cardíacas $(4 \% \times 12 \%)$. Quanto a mortalidade, cabe destacar que não houve henhum óbito na sala de operações no Grupo II e ocorreram três óbitos no Grupo I. Quando se analisa o tempo de ocorrência dos óbitos (imediato e hospitalar) e a causa (cardíaca e não cardíaca) existe maior prevalência de óbitos imediatos de causas cardíacas (6 casos) no Grupo I ( $p<0,01)$ em relação ao Grupo II (2 casos).

Dos cinco óbitos hospitalares ocorridos no Grupo II nenhum foi de causa cardíaca, três foram devido a síndrome da angústia respiratória do adulto (SARA) e dois devido a insuficiência de múltiplos órgãos (IMOS), o que pode ser associado a maior quantidade de líqüidos cristalóides utilizados neste grupo, já que não houve incidência destas complicações no Grupo I; esta conclusão pode ser corroborada pela maior necessidade de ventilação mecânica prolongada que ocorreu no Grupo II $(12 \% \times 6 \%)$.

Apesar destas diferenças, a análise estatística não demonstrou superioridade de um método sobre o outro, a não ser pelo fato dos pacientes do Grupo II terem tido um maior índice de recuperação espontânea ao ritmo sinusal $(p=0,032)$ e de ter havido maior incidência de óbitos imediatos de causas cardiacas no Grupo I ( $p<0,01)$. Estes dados, isoladamente, não nos autorizam a tirar conclusões, mas nos levam a acreditar que o retorno espontâneo ao ritmo sinusal pode estar associado à melhor proteção dos tecidos de condução e também melhor preservação da energia das células miocárdicas e que, apesar de não haver diferença estatística nos resultados referentes à morbi-mortalidade, a cardioplegia retrógrada foi mais eficaz na prevenção da síndrome de baixo débito pós-operatório e isto, provavelmente, interferiu na melhor evolução deste grupo.

\section{CONCLUSÕES}

Sabemos que comparar grupos experimentais, mesmo que semelhantes, mas com muitas variáveis, torna uma análise crítica definitiva quase impossível. Ainda em se tratando de um estudo clínico, onde as variáveis não são completamente dominadas, pequenas modificações podem ser infinitamente testadas, gerando milhões de cálculos e, conseqüentemente, várias conclusões diferentes.

A superioridade da cardioplegia retrógrada sobre a cardioplegia anterógrada, demonstrada neste estudo, não foi corroborada pela análise estatística. Ainda que conferindo ao Grupo retrógrada maior gravidade, o maior número de enxertos prejudicou a comparabilidade entre os grupos. O pequeno tamanho da amostra (100 pacientes) também foi determinante para estas conclusões. Isto nos leva a pensar que, se ampliássemos a amostra, a cardioplegia retrógrada poderia se mostrar mais efetiva na proteção miocárdica de pacientes portadores de miocardiopatia isquêmica grave.Esta impressão nos obrigou a adotá-la, como método de proteção miocárdica, neste grupo de pacientes em nosso Serviço. O aprimoramento da técnica e o reconhecimento de suas limitações diminuiu sobremaneira a ocorrência de complicações. 
Reis Filho FA R, Lima C M, Mota G G, Gonçalves LA, Gomes MC, Bernardes R C, Rabelo R C-Análise comparativa de dois métodos de proteção miocárdica em pacientes de alto risco para cirurgia de revascularização do miocárdio. Rev Bras Cir Cardiovasc $1996 ; 11(4): 238-47$.

RBCCV 44205-315

Reis Filho F A R, Lima C M, Mota G G, Gonçalves LA, Gomes M C, Bernardes R C, Rabelo R C-Comparative analysis of two methods of myocardial protection. Rev Bras Cir Cardiovasc 1996; 11 (4): 238-47 .

ABSTRACT: Objective: To compare the tradicional myocardial protection achieved by crystalloid cold cardioplegia, which is induced through the aortic root, associated to systemic hypothermia and intermitent aortic cross-clamping (Group I) to warm blood cardioplegia using induction through the aortic root but retrograde maintenance through the coronary sinus, associated to systemic normothermia.

Methods and Material: During the period ranging from May 1992 to May 1994, two groups of 50 patients were selected consecutively, but not randomized, according to requirements that increased the morbidity and mortality associated to the Coronary Artery Bypass Graft (CABG). All selected patients presented at the time clinical instability, more than three coronary arteries (or the left main coronary artery) occluded or severely suboccluded, associated with severe LV dysfunction of ischemic ethiology.

Results: The two groups were comparable according to the variabilities found in the period just prior to the surgery. Group II had a larger amount of grafts $(3.82 \times 3.40)$, consequently a longer period of aortic crossclamping ( $70.74 \times 62.06 \mathrm{~min})$ and a longer time in cardiopulmonary bypass (CPB) ( $97.5 \times 93.8 \mathrm{~min})$. Group I, in average, needed more inotropic agents for the weaning process from CPB, more use of mechanical assist devices and showed a bigger incidence of acute myocardial infarction as well as ventricular fibrilation after aortic cross-clamp release. This group also had less incidence of prolonged mechannical ventilation, greater early and global mortality, and also a larger number of deaths due to other cardiac causes.

Conclusion: The two groups were not comparable due to the larger number of grafts in Group II turning them more critical than Group I. Nervertheless, the results from Group II were superior to Group I, even though the statistic analysis do not corroborate those results. That, associated with clinical data, technical improvement and recognizing the limitations of the procedure, made us use it in this specific group of patients.

DESCRIPTORS: Heart arrest, induced, methods. Myocardial revascularization, risk factors. Myocardial revascularization, comparative study.

\section{REFERÊNCIAS BIBLIOGRÁFICAS}

1 Melrose D G, Dreyer B, Bentall H H, Baker J B E Elective cardiac arrest. Lancet 1955; 2: 21-2.

2 Blanco G, Adam A, Fernandez A - A direct experimental approach to the aortic valve: II. Acute retroperfusion of coronary sinus. J Thorac Surg 1956; 32: 171.

3 Gott V L, Gonzales J L, Zuhdi M N - Retrograde perfusion of coronary sinus for direct vision aortic surgery. Surg Gynecol Obstet 1957; 104: 319.

4 Lillehey C N, De Wall R A, Gott V L, Varco R L - The direct vision correction of calcified aortic stenosis by means of a pump-oxygenator and retrograde coronary sinus perfusion. Dis Chest 1956; 30: 123.

5 Solorzano J, Taitelbaum G, Chiu R C - Retrograde coronary sinus perfusion for myocardial protection during cardiopulmonary bypass. Ann Thorac Surg 1978; 25: 201-8.

6 Menasché $\mathrm{P}$, Kural $\mathrm{S}$, Fauchet $\mathrm{M}$ et al. - Retrograde coronary sinus perfusion: a safe alternative for ensuring cardioplegic delivery in aortic valve surgery. Ann Thorac Surg 1982; 34: 647-58.

7 Menasché $\mathrm{P}$ \& Pwinica A H - Retrograde coronary sinus perfusion. In: Roberts A J, ed. Myocardial protection during cardiac surgery. New York: Marcel Dekker, 1987: 251-62.

8 Shapira N, Lemole G M, Spagna P M, Bonner F J, Fernandez $\mathrm{J}$, Morse $\mathrm{D}$ - Antegrade and retrograde infusion of cardioplegia: assessment by thermovision. Ann Thorac Surg 1987; 43: 92-7.

9 Lazar H L - Coronary sinus interventions during cardiac surgery. Ann Thorac Surg 1988; 46: 475-82.

10 Chitwood W R - Myocardial protection by retrograde cardioplegia: coronary sinus and right atrial methods. In: Cardiac surgery: state of art reviews, vol.2. Philadelphia: Hanley and Belfus, 1988: 197-218.

11 Sutter F P, Goldman S M, Clancy M et al. - Continuous retrograde blood cardioplegia. Ann Thorac Surg 1991; 51: 136-7.

12 Bolling S F, Flaherty J T, Bulkley B H, Gott V L, Gradner $\mathrm{T} \mathrm{J}$ - Improved myocardial preservation during global ischemia by continuous retrograde coronary sinus perfusion. J Thorac Cardiovasc Surg 1983; 86: 659-66.

13 Gundry S R \& Hirsh M M - A comparison of retrograde versus antegrade cardioplegia in the presence of coronary artery obstruction. Ann Thorac Surg 1984; 38: $124-7$.

14 Horneffer P J, Gott V L, Gardner T J - Retrograde coronary sinus perfusion prevents infarct extension during intraoperative global ischemic arrest. Ann Thorac Surg 1986; 42:132-42.

15 Masuda M, Yonega K, Shiki K, Morita S, Kohno H, Tokunaga $\mathrm{K}$ - Myocardial protection in coronary occlusion by retrograde cardioplegic perfusion via the coronary sinus in dogs. J Thorac Cardiovasc Surg 1986; 92: 255-63. 
Reis Filho F A R, Lima CM, Mota G G, Gonçalves LA, Gomes M C, Bernardes RC, Rabelo R C-Análise comparativa de dois métodos de proteção miocárdica em pacientes de alto risco para cirurgia de revascularização do miocárdio. Rev Bras Cir Cardiovasc $1996 ; 11(4): 238-47$

16 Partington M T, Acar C, Buckberg G D, Julia P, Kofsky E R, Bugy H I - Studies of retrograde cardioplegia: I. Capillary blood flow distribution to myocardium supplied by open and occluded arteries. $J$ Thorac Cardiovasc Surg 1989; 97: 605-12.

17 Haan C, Lazar H L, Bernard S, Rivers S, Zallnick J, Shemin R J - Superiority of retrograde cardioplegia after acute coronary occlusion. Ann Thorac Surg 1991; 51: 408-12.

18 Schaper j, Walter $\mathrm{P}$, Scheld $\mathrm{H}$, Hehrlein $\mathrm{F}$. The effects of retrograde perfusion of cardioplegic solution in cardiac operations. J Thorac Surg 1985; 90: 882-7.

19 Snyder H E, Smithwick W, Wingard T, Agnew R C Retrograde coronary sinus perfusion. Ann Thorac Surg 1988; 46: 389-90.

20 Fiore A C, Naunheim K S, Kaiser G C et al. - Coronary sinus versus aortic root perfusion with blood cardioplegia in elective myocardial revascularization. Ann Thorac Surg 1989; 47: 684-8.

21 Lichtenstein S V, Ashe K A, Dalati H E, Cusimano R J, Panos A, Slutsky A S - Warm heart surgery. J Thorac Cardiovasc Surg 1991; 101: 269-74.

22 Buckberg G D - Antegrade/retrograde blood cardioplegia to ensure cardioplegic distribution: operative techniques and objectives. J Card Surg 1989; 4: 216-38.

Kalmbach T \& Bhayana J N - Cardioplegia delivery by combined aortic root and coronary sinus perfusion. Ann Thorac Surg 1989; 47: 316-7.

24 Drinkwater D C, Laks H, Buckberg G D - A new simplified method of optimizing cardioplegic delivery without righ heart isolation. J Thorac Cardiovasc Surg 1990; 100: 56-64.

25 Drinkwater D C, Cushen C K, Laks H, Buckberg G D - The use of combined antegrade/retrograde infusion of blood cardioplegic solution in pediatric patients undergoing heart operations. J Thorac Cardiovasc Surg 1992; 104: 1349-55.

26 Kennedy J W, Kaiser G C, Fisher L D et al. - Clinical and angiographic predictors of operative mortality from the Collaborative Study in Coronary Artery Surgery (CASS). Circulation 1981; 63: 793-802.

27 Naunheim K S, Fiori A C, Wadley S S - The changing profile of the patient undergoing coronary artery bypass surgery. J Am Coll Cardiol 1988; 11: 494-8.

28 Hearse D J, Braimbridge $M$ V, Jynge $P$ - Ischemia and reperfusion: the progression and prevention of tissue injury. In: Protection of the ischemic myocardium: cardioplegia. New York: Raven Press, 1981: 21.

Roberts A J - Myocardial protection in cardiac surgery. New York: Marcel Dekker, 1987.

30 Macmanus Q, Grunkemeier G, Lambert L, Dietl C, Starr A - Aortic valve replacement and aorta-coronary bypass surgery: results with perfusion of proximal and distal coronary arteries. J Thorac Cardiovasc Surg 1978; 75: 865-9.

31 Buckberg G D - A proposed "solution" to the cardioplegic

controversy. J Thorac Cardiovasc Surg 1979; 77: 803-15.

R C J, Blundell P E, Scott H J, Cain S - The importance of monitoring intramyocardial temperature during hypothermic myocardial protection. Ann Thorac Surg 1979; 28: 317-22.

Oldham $\mathrm{H} \mathrm{N}$, Jones R $\mathrm{H}$, Harris C C, Howe W R, Goodrich J k, Sabiston Jr. D C - Intraoperative relationships between regional myocardial distribution of bypass graft flow and the coronary collateral circulation. J Thorac Cardiovasc Surg 1979; 77: 32-8.

34 Hilton C J, Teubl W, Acker $M$ et al - Inadequate cardioplegic protection with obstructed coronary arteries. Ann Thorac Surg 1979; 28: 323-34.

35 Fishman $\mathrm{N} \mathrm{H}$ \& Abouav $\mathrm{J}$ - Myocardial temperature differences as a guide to the order of coronary artery bypass anastomoses in high-risk patients. $A m ~ J$ Surg 1980; 140: 92-8.

36 Ekroth R, Berggren H, Sudow G, Wojciechowski J, Zackrisson B F, Willian-Olsson G - Thermographic demonstration of uneven myocardial cooling in patients with coronary lesions. Ann Thorac Surg 1980; $29: 341-5$

37 Daggett W M, Jacocks M A, Coleman W S, Jonhson R G, Lowenstein E, Vander Salm T J - Myocardial temperature mapping: improved intraoperative myocardial preservation. J Thorac Cardiovasc Surg 1981; 82: 883-8.

38 Becker H, Vinten-Johansen J, Buckberg G D, Follette D M, Robertson J M - Critical importance of ensuring cardioplegic delivery with coronary stenosis. $J$ Thorac Cardiovasc Surg 1981; 81: 507-15.

39 Grodin C M, Helias J, Vonhe P R, Robert P - Influence of a critical coronary artery stenosis on myocardial protection through cold cardioplegia. $J$ Thorac Cardiovasc Surg 1981; 82: 608-12.

40 Landymore R W, Tice D, Trehan N, Spencer F . Importance of topical hypothermia to ensure uniform myocardial cooling during coronary artery bypass. $J$ Thorac Cardiovasc Surg 1981; 82: 832-6.

41 Heineman F W, MacGregor D C, Wilson J G, Ninomiya $\mathrm{J}$ - Regional and transmural myocardial temperature distribuition in cold chemical cardioplegia. J Thorac Cardiovasc Surg 1981; 81: 851-9.

42 Robertson J M, Buckberg G D, Viten-Johansen J, Leaf J D - Comparison of distribution beyond coronary stenosis of blood and asanguineous cardioplegic solutions. Thorac Cardiovasc Surg 1983; 82: 80-6.

43 Rosenkranz E R, Vinten-Johansen J, Buckberg G D, Okanoto F, Edward H, Bugyi $H$ - Benefits of normothermic induction of blood cardioplegia in energy-depleted hearts, with maintenance of arrest by multidose cold blood cardioplegia infusions. $J$ Thorac Cardiovasc Surg 1982; 84: 667-77.

44 Teoh K H, Christakis G T, Weisel R D - Accelerated myocardial metabolic recovery with terminal warm blood cardioplegia (hot shot). J Thorac Cardiovasc Surg 1986; 91: 888-95. 